Bernhard J. Dotzler, Henning Schmidgen (Hg.)

\title{
Parasiten und Sirenen
}

Zwischenräume als Orte der materiellen Wissensproduktion 
Bernhard J. Dotzler, Henning Schmidgen (Hg.) Parasiten und Sirenen

Literalität und Liminalität hrsg. v. Achim Geisenhanslüke und Georg Mein | Band 6 
Bernhard J. Dotzler (Dr. phil.) ist Professor für Medienwissenschaft an der Universität Regensburg. Sein Arbeitsschwerpunkt liegt auf dem Gebiet der Medialitätsforschung unter besonderer Berücksichtigung der Geschichte digitaler Medien und der Schnittstellen zwischen Medien- und Wissenschaftsgeschichte.

Henning Schmidgen (Dr. phil.) ist wissenschaftlicher Mitarbeiter am Max-Planck-Institut für Wissenschaftsgeschichte, Berlin. Schwerpunkte seiner Tätigkeit sind die Geschichte experimenteller Lebenswissenschaften, das Verhältnis von Zeit und Wissen sowie die Entwicklung Virtueller Laboratorien. 
Bernhard J. Dotzler, Henning Schmidgen (Hg.)

Parasiten und Sirenen.

Zwischenräume als Orte der materiellen Wissensproduktion 
Die Übersetzungen im vorliegenden Band wurden im Rahmen des Programms »Schlüsselthemen der Geisteswissenschaften « durch die Volkswagenstiftung, Hannover, gefördert. (Projekt: »Die Experimentalisierung des Lebens«, MPIWG)

Bibliografische Information der Deutschen Nationalbibliothek Die Deutsche Nationalbibliothek verzeichnet diese Publikation in der Deutschen Nationalbibliografie; detaillierte bibliografische Daten sind im Internet über http://dnb.d-nb.de abrufbar.

(C) 2008 transcript Verlag, Bielefeld

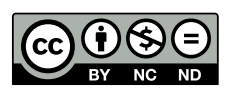

This work is licensed under a Creative Commons Attribution-NonCommercial-NoDerivatives 3.0 License.

Umschlaggestaltung: Kordula Röckenhaus, Bielefeld Umschlagabbildung: Resonator aus Glas, entnommen aus: Hermann von Helmholtz, Die Lehre von den Tonempfindungen als physiologische Grundlage für die Theorie der Musik. 3. Aufl., Braunschweig: Vieweg, 1870. S. 73, Abb. I6a

Satz: Christine Fraunhofer

Druck: Majuskel Medienproduktion GmbH, Wetzlar ISBN 978-3-89942-870-4

Gedruckt auf alterungsbeständigem Papier mit chlorfrei gebleichtem Zellstoff.

Besuchen Sie uns im Internet: http://www.transcript-verlag.de

Bitte fordern Sie unser Gesamtverzeichnis und andere Broschüren an unter: info@transcript-verlag.de 


\section{INHALT}

\section{Einleitung \\ Zu einer Epistemologie der Zwischenräume \\ BERNHARD J. DOTZLER \& HENNING SCHMIDGEN \\ 7}

\section{Parasitäre Verhältnisse}

Wirt oder Gast?

Interaktionen zwischen Literaturkritik, experimenteller Psychologie und Medizin, 1890-1910

MARIE GUTHMÜLLER

21

Der Löwe kommt

Nervöse Topologien bei Angelo Mosso

PHILIPP FELSCH

49

Frauen am Rande der Datenverarbeitung Zur Produktionsform einer Weltgeschichte der Technik MARKUS KRAJEWSKI

63

\section{Bioskopien}

Die Geschwindigkeit des Empfindens Philosophie im Zeitalter der Bewegungstechnologien JIMENA CANALES

83

Leerstellen des Denkens

Die Entdeckung der physiologischen Zeit

HENNING SCHMIDGEN 


\section{PARASITÄRE VERHÄLTNISSE}





\section{Frauen AM RANDE DER DATENVERARBEITUNG ZUR PRODUKTIONSFORM EINER WELTGESCHICHTE DER TECHNIK MARKUS KRAJEWSKI}

Ich bin die Ordnung in Person. Franz Maria Feldhaus

\section{Das Resultat}

Was haben eine Säge, ein Bleistift und eine Badewanne gemeinsam? Nein, nicht etwa dass es sich hier um eine in den Begriffen verwechselte, durch Lautréamont berühmt gewordene, inzwischen jedoch längst überstrapazierte Begegnung präsurrealistischer Art handelte. ${ }^{1}$ Wenngleich sich Regenschirm, Nähmaschine und Seziertisch ebenso an jenem Ort einfinden, wo Bleistift, Säge und Badewanne nebst zahllosen anderen Gegenständen wie beispielsweise Drahtseilschwebebahnen, Schläuche, Funkentelegraphen, Wärmflaschen, Lötkolben oder Altmeister des Segelflugs zusammentreffen. Die Gemeinsamkeit all dieser Gegenstände besteht darin, dass sich zwar weniger die Objekte selbst, dafür jedoch die Geschichten ihrer Herkunft, Erfindung und Entstehungsweise in einer einzigen Quelle versammelt finden, und zwar in der stattlichen Sachkartei der sog. Quellenforschungen zur Geschichte der Technik und Indus trie G.m.b.H. von Dr. Ing. h.c. Franz Maria Feldhaus.

Mit dem Anfang des 20. Jahrhunderts beginnt Franz Maria Feldhaus (1874-1957), jedes Datum und kleinste Fragment, das »irgendwie technisch-historisch aussieht «, ${ }^{2} \mathrm{zu}$ sammeln und akribisch $\mathrm{zu}$ archivieren. Nach der ersten Hälfte des Jahrhunderts wird diese Sammlung auf ca. 160.000 Einträge angewachsen sein, säuberlich verzeichnet auf Kar-

1 Der Held der Geschichte ist schön »wie die unvermutete Begegnung einer Nähmaschine und eines Regenschirms auf einem Seziertisch!«, Lautréamont: Die Gesänge des Maldoror, Reinbek bei Hamburg 1990, S. 223.

2 Franz Maria Feldhaus: »Archiv >Geschichte d. Technik, e.V.«, in: Geschichtsblätter für Technik, Industrie und Gewerbe (1927a), Bd. 11, Nr. 2, S. 158. 
teikarten, funktional ausdifferenziert, rubriziert und unterteilt in die "große Sachkartei« (71.000 Karten), die »kleinere Personenkartei« (24.000), »Jahreskartei« (15.000), »Tagesdatenkartei« für den Kalender (12.000, Besonderheit: 12 Schiebeladen für 12 Monate), »Kartei der technischen Sprüche« (2.400 Einträge), ein Archiv u.a. mit Fragebogendaten und allerhand anderem Schriftgut - sowie der »Kartei der eigenen Publikationen« (ca. 5200 Karten), ${ }^{3}$ wo selbstverständlich auch die passenden Monographien zu den oben aufgezählten Gegenständen nachgewiesen sind. Daneben verzeichnet diese Kartei zweiter Ordnung, die schließlich all das verzeichnet, was zuvor als Text aus den Fragmenten des Karteifundus hervorging, 36 weitere eigenständige Monographien zu durchaus ungewöhnlichen oder abseitigen Themen ${ }^{4}$ der Technikgeschichte sowie 3807 Artikeln aus Feldhaus' eigener Hand. Darunter befinden sich etwa Abhandlungen über »Techniker in Walhalla«, die Aufhebung der Schwerkraft, Reiseblitzableiter, Mondtelegraphie, Mäusekraft oder Salatmaschinen. Nicht fehlen in dieser kleinen Inventarsliste der »Sammlung Feldhaus« dürfen überdies die Bildabteilung mit ihren drei Untergruppen sowie die Technische Medaillensammlung.

Als jemand, der mehr als fünfzig Jahre Tag für Tag an der Erweiterung und Vervollständigung dieser Datensammlung gearbeitet hat, bemisst Feldhaus den Wert seines zusammengetragenen Materials kaum überraschend mit einem denkbar kostbaren Kriterium. Lakonisch bemerkt er, dass keine Feuerversichungsgesellschaft das Risiko zu tragen

3 Die Bezeichnung der einzelnen Karteien folgt Franz Maria Feldhaus: »Die Sammlungen Feldhaus und ihr neues Heim«, in: Geschichtsblätter für Technik, Industrie und Gewerbe (1927b), Bd. 11, Nr. 1, S. 6-12, hier S. 6f, vgl. ergänzend dazu, in leicht variiertem Wortlaut sowie mit aktualisierten statistischen Angaben des Kartei-Systems Wolfgang Kohte: »Technikgeschichtliche Quellenerschließung im Feldhaus-Archiv, in: Der Archivar. Mitteilungsblatt für deutsches Archivwesen, Bd. 9, Nr. 3, S. 206-209. Die statistischen Daten sind auf dem Stand von 1956, also ein Jahr vor Feldhaus' Tod, leicht nach unten korrigiert. Zwischenzeitlich (1927) veranschlagt Feldhaus den Umfang der Karteien weitaus höher; so beziffert er die Einträge der Sachkartei »mit etwa 80000 Karten, von denen jede nur ein einziges Datum enthält.« (S. 8). Zum Ende des Zweiten Weltkriegs sollen jedoch - nach Auskunft seiner Tochter Eva Zeller - einige Karten dem unmäßigen Hunger Kasseler Mäuse zum Opfer gefallen sein.

4 Herausragend in dieser Kollektion bleibt sicherlich die Kulturgeschichte des Abtritts, die von antiken Toiletten bis hin zum automatischen Wasserklosett reicht, erschienen unter dem Akronym $\mathrm{Ka}-\mathrm{Pi}-\mathrm{Fu}$ und andere verschämte Dinge. Ein fröhlich Buch für stille Orte, wobei die drei Silben für jeweils eine Hervorbringung des menschlichen Verdauungssystems stehen. 
bereit sei, im Fall eines Verlusts eine adäquate Summe zu zahlen. ${ }^{5}$ Eine würdigende Einschätzung erhält die Sammlung 1953 derweil vom Direktor des noch jungen Bundesarchivs in Koblenz. Die »Forschungsstelle für Geschichte der Technik und Industrie« besitze »in Deutschland kaum ihresgleichen «. ${ }^{6}$ Als besonderes Kennzeichen hebt Winter nicht zuletzt »die straffe Organisation der Forschung« hervor, um zu resümieren: »Diese Sammlungen sind in folgerichtiger Entwicklung einer klar erkannten Leitidee als Lebenswerk aufgebaut «. ${ }^{7}$

Was sich hinter dem Merkmal einer straffen Organisation in diesem Fall verbirgt, welches Produktionsarrangement, welche medientechnische Implementierung und historiographische Methodik dieser Leitidee, dem Versuch also, eine möglichst vollständige »Weltgeschichte der Technik « ${ }^{8}$ aufzuschreiben, zugrunde liegt, soll im Folgenden eine nähere Untersuchung erfahren. Bei diesem - vorsichtig formuliert großzügig konzeptionierten Projekt galt es, viele Kämpfe an mehr als einer Front auszufechten und dabei zahlreichen Widerständen zu begegnen, zu deren erfolgreicher Überwindung oder schlussendlich gescheiterter Umsetzung eine eingehende Analyse bislang fehlt. Doch zuvor sei ein Blick auf die Persönlichkeit Franz Maria Feldhaus gestattet, auf die Umstände und Kontexte, die ihn zum seinerzeit keinesfalls unumstrittenen, heute indes nahezu vergessenen »Nestor der deutschen Technikhistoriker « ${ }^{9}$ machten.

\section{Die (Ordnung in) Person}

Franz Maria Feldhaus ist Autodidakt. Seine Biographie, insbesondere der frühen Jahre, liegt weitestgehend im Dunkeln. Diejenigen Details, die bekannt sind, bleiben zudem nicht frei von Stilisierung und Selbstmystifizierung. In aller Kürze seien einige Stationen seiner Biographie aufge-

5 Franz Maria Feldhaus: »Geschichte der Technik«, in: Geschichtsblätter für Technik, Industrie und Gewerbe (1927), Bd. 11, Nr. 1, S. 10.

6 Dr. Winter: »Betr.: Feldhaus-Archiv in Wilhelmshaven-Altengroden«, in: Bundesarchiv (1953), Nr. Az. 206, S. 1.

7 Ebd.

8 Anonym: »Persönliche Nachrichten - Franz Maria Feldhaus 80 Jahre«, in: Gas- und Wasserfach (1954), Bd. 95, Nr. 12, S. 399.

9 Carl Graf von Klinckowström: »Nachruf auf Franz Maria Feldhaus«, in: Archives Internationales d'Histoire des Sciences (1957), Bd. 10, Nr. 40, S. 303f., hier S. 303. 
listet: ${ }^{10}$ Geboren 1875 in Neuß/Rheinland verlebt Feldhaus eine wechselvolle Jugend, die unter anderem aus Episoden als Jesuitenschüler, Gelegenheitsarbeiter, Monteur, Schreiber, Handlungsreisender, Kleinunternehmer in Sachen Elektro-Installation besteht sowie in einem Überwachungsposten eines Kraftwerks bei Rheinfelden, Schweiz. Ab 1900 beginnt seine Beschäftigung mit Technikgeschichte, die Feldhaus seinerzeit als terra incognita, als weitestgehend unbeschriebenes Blatt einstuft. 1904 lebt er in Heidelberg, wo seine Tätigkeit als Autor der Technikgeschichte einsetzt mit Studien zum Erfinder der elektrischen Verstärkungsflasche Ewald Jürgen von Kleist und über den Entdecker des Erdmagnetismus William Gilbert. Feldhaus' persönliches Gründungsdokument für eine deutsche Technikgeschichtsschreibung stellt jedoch das ebenfalls 1904 erscheinende Lexikon der Erfindungen und Entdeckungen auf den Gebieten der Naturwissenschaften und Technik in chronologischer Übersicht dar.

Das Lexikon erfährt rasch die nächste Ausbaustufe mit einer Erweiterung zu Feldhaus' Buch der Erfindungen von 1908. Diese Arbeit spielt wohl kaum zufällig auf das nur wenige Jahre zuvor in neunter Auflage erschienene Buch der Erfindungen an. Es zeigt jedoch deutlich mit der Nennung des Eigennamens im Titel, dass es sich hier weniger um eine Weiterführung oder Variante des seit 1854 erscheinenden, zuletzt von Franz Reuleaux, dem renommierten Charlottenburger Physiker, und anderen verantworteten Nachschlagewerks handelt, sondern statt dessen einen eigenen Standard zu setzen beabsichtigt, um mit dem »längst überholten Stand des Wissens « ${ }^{11}$ aufzuräumen.

Doch sieht man hier bereits, dass sich das Feld der Geschichte von Technik und Industrie so ganz unerkundet nicht ausnimmt wie Franz Maria Feldhaus es gerne hätte. Allen voran stehen Studien von Theodor Beck zur Geschichte des Maschinenbaus sowie darüber hinaus Schriften seines Bruders Ludwig zur Geschichte des Eisens in technischer und kulturgeschichtlicher Beziehung. Auch Conrad Matschoß, mit dem Feldhaus nicht nur Konkurrenz, sondern eine regelrechte Erzfeindschaft verbindet, legt seine Geschichte der Dampfmaschine bereits 1901 vor. Daneben gibt es weitere Versuche, mit großangelegten Projekten aktuelle Übersichten zur Technik und ihrer Geschichte herzustellen, etwa - einmal mehr - von einem Beck, diesmal Hermann, der ab 1908 in Berlin das Institut für Techno-Bibliographie aufzubauen beginnt. ${ }^{12}$

$10 \mathrm{Zu}$ ausführlicheren Informationen vgl. Markus Krajewski: Restlosigkeit. Weltprojekte um 1900, Frankfurt a. M. 2006, Kap. 3.

11 C. Graf von Klinckowström: »Nachruf auf Franz Maria Feldhaus«, S. 303.

12 Vgl. dazu Thomas Hapke: »Wilhelm Ostwald, the Brücke (Bridge), and Connections to Other Bibliographic Activities at the Beginning of the 
Was alle Vor-, Mit- oder Gegenläufer im Unterschied zu Feldhaus eint, bleibt ihre akademische Ausbildung, die stets in direktem Zusammenhang mit Maschinenbau oder Ingenieurskunst steht. Franz Reuleaux, Theodor Beck, Conrad Matschoß et al. sind ausnahmslos kraft ihrer Profession Techniker oder Ingenieure und bekleiden größtenteils entsprechend repräsentative Ämter an den Universitäten bzw. in professionellen Berufsvereinigungen wie dem Verein Deutscher Ingenieure (VDI). Feldhaus hingegen ist weder Techniker noch Historiker, weder Mitglied einer Universität (je gewesen) noch einer lobbyistischen Vereinigung. Mit den Geschichtswissenschaften verbindet ihn vor allem methodisch wenig. ${ }^{13}$ Der vormalige Gelegenheitsarbeiter, Monteur, Elektro-Installateur und Kleinunternehmer ist Autodidakt in wissenschaftlichen Großprojekten und kraft eigener Bezeichnung »Ingenieur«. ${ }^{14}$

Auf der anderen Seite war Feldhaus jedoch auch - gemäß den Beschreibungen von Eva Zeller, seiner Tochter aus dritter Ehe - »Kanaille«, »Lebemann«, »unglückseliges Menschenkind« bis hin zum »mörde-

Twentieth Century, in: Mary Ellen Bowden, Trudi Bellardo Hahn und Robert V. Williams (Hg.), Proceedings of the 1998 Conference on the History and Heritage of Science Information Systems, ASIS Monograph, Information Today, Medford, NJ, S. 139-147. Becks Institut verortet sich allerdings eher im Umkreis der Bibliographie-Bewegung um 1900, zu dem auch und vor allem die deutschen Ableger des Institut International de Bibliographie aus Brüssel, d.h. Wilhelm Ostwald und sein Institut zur Organisierung der geistigen Arbeit namens Die Brücke zählt, siehe dazu auch Markus Krajewski: Zettelwirtschaft. Die Geburt der Kartei aus dem Geiste der Bibliothek, Berlin 2002, Kapitel 6. In einer Würdigung von Theodor Beck anläßlich seines zehnten Todestages verwechselt Feldhaus im ersten Satz bemerkenswerterweise die Vornamen: statt »Theodor« taucht hier »Hermann« auf, der ansonsten als Leiter eines Konkurrenzunternehmens geflissentlich keine Erwähnung findet, vgl. Franz Maria Feldhaus: »Theodor Beck †«, in: Geschichtsblätter für Technik, Industrie und Gewerbe (1917), Bd. 4, Nr. 1-12, S. 161f., hier S. 161.

13 Offenbar haben (akademische) Historiker - trotz Johannes Beckmanns einstiger Konturierung der Disziplin unter dem Namen Technologie mit engen Verflechtungen $\mathrm{zu}$ den nachmaligen sog. Geisteswissenschaften immer wieder vor einer eingehenden Beschäftigung mit Technik in ihrer historischen Genese zurückgescheut. Der Mangel an Beispielen für die Annäherung seitens der allgemeinen Historiographie an das Feld der Technik- und Ingenieurswissenschaften muß hier allerdings eine offene Frage bleiben.

14 Vgl. etwa das Vorwort zur ersten Auflage von Die Technik der Vorzeit, der geschichtlichen Zeit und der Naturvölker von 1914. 
rischen Liebesstümper «. ${ }^{15}$ Diese auf den ersten Blick wenig relevante, weil private Tätigkeit spielt, wie noch zu zeigen sein wird, so extrem in die Produktionsbedingungen des Großprojekts Weltgeschichte der Technik hinein, dass sie nahezu als konstitutiv bilanziert werden muß.

\section{Registraturen}

\subsection{Frauen}

Wenn im Aufschreibesystem 1900 die Unordnung des Autorenschreibtischs die maschineschreibende Hand einer Frau zu Hilfe und zur Ordnung ruft, und die zu dieser Hand gehörende Frau zwar keine Liebe, dafür aber rund 200 Silben pro Minute schafft, ${ }^{16}$ so benötigt auch Franz Maria Feldhaus diese Triebkraft für sein Lebenswerk. Demzufolge befindet er sich auf der steten Suche nach Kleio, seiner Muse der Historiographie, um sie als Herodot der Technik - das Brautgeschenk ist sein Projekt selbst - zu werben. Dabei macht er augenscheinlich eine gute Figur, wie die zahlreichen Photographien belegen, die er von sich selbst inszeniert. Das Photolabor im eigenen Haus ist von Kopf bis Fuß auf Feldhaus eingestellt. Seine Tochter Eva-Maria erinnert sich:

»[H]ier sind die Wände von oben bis unten mit Fotos tapeziert, die E-M nicht sehen will: hundertmal Vater, lesend, schreibend am Schreibtisch, rauchend im Sessel, lachend, die Zunge rausstreckend, die Augen mit der Hand beschattend, Kopfbild, Brustbild, in voller Größe, von vorn, von der Seite, vor seinen Karteikästen, vor Bücherreihen, Vater in Badehose vor Meeren, in Strandkörben, eine Dame im Arm, in Sandburgen sich aalend, Vater, den Namen FRANZ mit Muscheln in den Sand legend, Vater in Zylinder, im Tropenhelm unter Palmen, im Südwester, zwei Damen im Arm, vor Ruinen ausgegrabener Städte, Vater nebem einem Gipsrelief seiner selbst, Vater mit Esther, Julia, mit anderen Babies, altmodischen in Steckkissen und Häubchen, Vater huckepack auf dem Rücken einer fülligen Dame, Vater mit Damen, mit Hunden, allein vor seiner hochrückigen Schreibmaschine. $\ll{ }^{17}$

Nicht nur die Damen vermehren sich, erst eine, dann zwei, zunächst nur im Arm, schließlich trägt frau ihn samt dem Lebenswerk. Und die Serie

15 Eva Zeller: Solange ich denken kann. Roman einer Jugend, Stuttgart 1981, S. 59f, 112, oder Eva Zeller: Nein und Amen. Autobiographischer Roman, Stuttgart 1986, S. 194.

16 Vgl. Friedrich Kittler: Aufschreibesysteme 1800 • 1900, 3., vollständig überarbeitete Aufl., München 1995, S. 463.

17 E. Zeller: Solange ich denken kann, S. 77. 
der Damen bringt eine Serie von Kindern hervor, die zwar nicht im Arbeitszimmer spielen dürfen, dafür dortselbst aber später arbeiten sollen. Es ertönt die Symphonie aus drei Typographien/Schreibmaschinen. EvaMaria Zeller hört als Kind von »oben die Schreibmaschinen von Vater und Vaters Frau und Vaters Sekretärin klappern «. ${ }^{18}$

Diese Konstellation stabilisiert sich schließlich mit der letzten Ehe. Nummer vier in der Serie heißt Margarete - genau wie ihre Vorvorgängerin. Und genau wie jene arbeitete diese Margarete zuvor als Büroangestellte am Projekt Weltgeschichte. Das Bildungsgesetz zur Rekrutierung der »Bürohilfskräfte« gleich Ehefrauen genügt schlicht einem Statuswechsel und einer Änderung der Verfügungsgewalt von bezahlter Teilzeitsekretärin zu unbezahlter Vollzeitgattin, mit allen Rechten und Pflichten. 1923, es läuft gerade Ehe Nummer drei mit Elisabeth, steht das »Forschungsheim für die Geschichte der Technik» kurzzeitig in Eberswalde, wo auch Eva-Maria Zeller, geb. Feldhaus, zur Welt kommt. Von dem Haus gibt es eine Photographie: »Aus fast jedem Fenster schaut eine Dame. Harem mit meinen Lieblingsfrauen steht in Vaters Schrift auf der Rückseite der Aufnahme. « ${ }^{19}$

\subsection{Und Kinder zuerst}

Sofern in dem Haus in Eberswalde, wie bei anderen Stationen der Odyssee des Forschungsheims auch, die Produktion von Technikgeschichten und Ehen zusammenfallen, bleiben die Ergebnisse in Form von zahlreichen neuen Karteikarten - und Kindern nicht aus. Beide Resultate dienen als Bausteine zur Konstruktion und Sicherung des Lebenswerks. Das Projekt funktioniert generationenübergreifend. Der Sohn aus erster Ehe, Gilbert, ${ }^{20}$ ist im Familienbetrieb ein ebenso notwendiger Bestandteil wie die Gattin oder Sekretärin. Wenngleich Gilbert unter Dysfunktionalitäten leidet. Eine angeborene Krankheit läßt seine Hände immerzu zittern; ein Problem, das sich im Rahmen der Mitarbeit am Bürobetrieb des Vaters nur noch verschlimmert, ${ }^{21}$ was ihn nicht zuletzt vor einige Schwierigkeiten bei der täglichen Arbeit des Ordnens und Schreibens gestellt haben dürfte. So erklärt sich auch sein ebenso praktischer wie mitleiderregender

18 Ebd., S. 69.

19 Ebd., S. 248.

20 Benannt nach dem Subjekt aus Feldhaus zweiter Publikation William Gilbert, vgl. Franz Maria Feldhaus: Die Begründung der Lehre von Magnetismus und Elektrizität durch Dr. William Gilbert, Heidelberg 1904.

21 Vgl. E. Zeller: Solange ich denken kann, S. 405. 
Bericht über das »Richtige Abstellen von Kartothekkarten«, der 1929 in der Zeitschrift für Organisation erscheint. $^{22}$

Gleichzeitig gilt es, die weiteren Nachkommen auf das Lebensprojekt auszurichten. Von Beginn seiner Sammlertätigkeit an erachtet Feldhaus die Frage nach der Zukunft und dem Fortbestand der Sammlung als höchst dringend. »Schon kurz vor dem Weltkrieg war die Frage für mich und meine Mitarbeiter, Margarete Feldhaus und Graf Klinckowstroem, brennend, was einstmals aus alldem Zusammengetragenen werden sollte. ${ }^{23}$ Schon früh sieht Feldhaus demzufolge auch seine Tochter EvaMarie - wie später die Töchter Herrad (mit Erfolg) und Barbara Angela (weniger erfolgreich) - als Mitarbeiterin an den Karteikästen vor, um die Kontinuität und den Ausbau der Sammlung langfristig zu sichern. »Alle seine Frauen hat er dorthin gesetzt, alle Töchter, sofern sie sich setzen ließen, und unerbittlich den Sohn Gilbert, bis dem der Arm zuckte und zuckte, und er vor lauter Mitarbeit zu gar nichts anderem kam als zu einem qualvollen Hickhack mit seinem Vater «. ${ }^{24}$ Doch Eva-Marie widersetzt sich diesen Plänen von »[s]einer Machtergreifung ${ }^{25}$ nachhaltig und mit Erfolg. Auch die Strategie, die Zahl weiterer potentieller Nachfolger kurzerhand durch neue Kinder zu erweitern, erweist sich nicht immer als praktikabel. Denn »[s]eine Sekretärin habe ihm einen geistig behinderten Sohn Ferdinand geboren. $\ll^{26}$ So greift der Vater im Fall von Eva-Marie schließlich zu unorthodoxen Methoden, ihre Unselbständigkeit zu erhalten, um sie doch noch auf sein Lebenswerk zu verpflichten. Ihr Studium an der Universität Greifswald und allen anderen Hochschulen soll durch sein Veto unterbunden werden. »Hiermit beantrage ich, meine Tochter Eva-Marie von allen deutschen Universitäten auszuschließen. Hochachtungsvoll. ${ }^{27}$ Doch abgesehen von der jüngeren Tochter Herrad $^{28}$ und $»$ seiner treuen Mitarbeiterin Margarete ${ }^{29}{ }^{29}$ gelingt es Feldhaus letztlich nicht, die familiäre Kontinuität des Sammlungspro-

22 Gilbert Feldhaus: »Richtiges Abstellen von Kartothekkarten«, in: BüroOrganisation (1929), Bd. 3, Nr. 2, S. 15.

23 F. M. Feldhaus, »Geschichte der Technik«, S. 2.

24 E. Zeller: Solange ich denken kann, S. 405, vgl. auch S. 321.

25 Ebd., S. 335.

26 Vgl. etwa ebd., S. 329.

27 Ebd., S. 225.

28 Benannt nach der Äbtissin zu Hohenburg Herrad von Landsberg, die im 12. Jhd. in ihrer Schrift Garten des Vergnügens durch technische Zeichnungen hervortrat.

29 Carl Graf von Klinckowström: Franz Maria Feldhaus, der Historiker der Technik, o.O. 1924, o.S. 
jekts, »dessen Sicherung über seinen Tod hinaus seine letzte Sorge war «, ${ }^{30} \mathrm{zu}$ gewährleisten.

»Wenn der Begriff Geschichte irgendeinen Sinn hat, dann beschreibt er doch wohl die Struktur und Zerstörung menschlicher Beziehungen. ${ }^{31}$ Heiner Müllers Diktum ließe sich ebenso umgekehrt auf Franz Maria Feldhaus anwenden. Denn dessen Sinn folgt einzig einer Beschreibung der Geschichte der Technik, die einer Struktur und Zerstörung der familiären Beziehungen aufruht. Feldhaus führt einen Mehrfrontenkrieg, nach außen gegen eine sich unabhängig von ihm etablierende Technikgeschichte, nach innen für die Sicherung der Sammlung Feldhaus. Weitestgehend unberücksichtigt in der geplanten Kontinuität der Sammlung bleiben dabei allein die unehelichen Kinder, deren Zahl bis heute nur schwierig zu bestimmen ist. »Unser Held bemerkte, daß er durch den häufigen Besuch von Höhlen [...] die Regeln der Logik übertrat und einen Circulus vitiosus beging. $\ll^{32}$

\section{Unsichtbare Dritte: Parasiten, Dilettanten}

>Unser Held<, Franz Maria Feldhaus, hat im Laufe seines Lebens ein weit verzweigendes, in seiner Art einzigartiges Archiv zu nahezu jedem kleinstem Detail und Datum der Technikgeschichte versammelt, ein systematisch und bibliothekstechnisch nach allen Regeln der Kunst differenziertes Karteisystem, auf dessen Grundlage tausende von Aufsätzen, Zeitschriftenbeiträge, hunderte von Monographien - veröffentlichte ebenso wie unpublizierte -, Firmenschriften, Lexika, Kalendergeschichten usw. entstanden sind. Zusammenstellung und Anhäufung dieser Quellenstudien folgen dabei einer spezifischen, durchaus als unkonventionell zu charakterisierenden Methode, die Feldhaus infolge seiner autodidaktischen Entwicklung zum Technikhistoriker dazu veranlaßt, die gängigen wissenschaftlichen Kriterien für seine eigenen Zwecke zu adaptieren. Gleichzeitig erfordert das auf Vollständigkeit angelegte Konzept einer Weltgeschichte der Technik ein besonderes Aufschreibeverfahren, das alle zur Verfügung stehenden Ressourcen einbeziehen muß. Feldhaus betreibt ein System, das neben der Rezeption und Inkorporation aller erreichbaren Fakten der Technikgeschichte vor allem die eigenen familiären Verhältnisse zur Hervorbringung von Texten einzuspannen versteht.

30 C. Graf von Klinckowström: »Nachruf auf Franz Maria Feldhaus«, S. 303.

31 Heiner Müller: Gesammelte Irrtümer 3. Texte und Gespräche, Theaterbibliothek, Frankfurt a. M. 1994, S. 152.

32 Lautréamont: Die Gesänge des Maldoror, S. 220. 
Das eingangs beschriebene Resultat, die einstmals 160.000 Karteikarten umfassende Datenbank der Technikgeschichte, bildet dabei das Zentrum der Familienproduktion, und zwar im doppelten Sinn: zum einen versucht Feldhaus über seine jeweiligen Lebensabschnitte hinweg, die familiäre Konstellation konsequent auf das Lebenswerk auszurichten, also Ehefrau und Kinder im arbeitsfähigen Alter fortwährend in die Arbeit an der Kartei einzubinden - wobei die vormaligen Mitarbeiterinnen oder Sekretärinnen nicht nur einmal aufrücken in die Position der Gattin. Die Objektwahl, um mit Theweleit zu sprechen, ${ }^{33}$ genügt also streng den psychotechnischen Kriterien einer büroorganisatorischen Eignung und Verwendungsfähigkeit. Zum anderen dienen einzig die Kartei und ihre textuellen Hervorbringungen zur Sicherung des Lebensunterhalts für die gesamte Familie. Die kontinuierliche Versammlung von Karteikarten gerät zur exklusiven Existenzbedingung, die es demzufolge nach >außen abzusichern gilt, denn ihr Fortbestehen und eine ertragreiche Ausbeute muß zu jedem Zeitpunkt gesichert bleiben. Diese Grundkonstellation bringt einige Effekte und funktionale Voraussetzungen mit sich. Feldhaus muß - wenn diese Übertragung des Begriffs der Kulturtechnik im seinerzeit üblichen Sinn von Agrikultur und Melioration (Bewässerungstechnik) gestattet sei - ein neues Feld bestellen.

Zunächst steht Feldhaus jedoch noch in der Verlegenheit, sich einen Namen zu machen, als Herodot der Technik bekannt zu werden, das Wissen um eine Anlaufstelle für Technikhistörchen zu lancieren und zu verbreiten, eine Zentrale zu bilden, an der Informationen zu jeweiligen Fachfragen zuverlässig eingeholt werden können. Diese Etablierung eines Markennamens Feldhaus kann dabei nicht auf die Hilfe üblicher Institutionen zurückgreifen. Denn einerseits mangelt es Feldhaus an einer entsprechenden akademischen Ausbildung und damit an ersten Anknüpfungspunkten. Andererseits schlagen die gelegentlich in Angriff genommenen Kooperationen mit herkömmlichen akademischen Forschungsstätten einer sich gerade erst herausbildenden Technikgeschichte fehl. Die Versuche, seine Quellenforschungen mit anderen Institutionen zu koppeln, scheitern ausnahmslos und hinterlassen nicht selten >verbrannte Erde`, so dass schließlich nur der Ausweg bleibt, die eigene Unternehmung gegen alle Widerstände selbst zu institutionalisieren.

Feldhaus akzeptiert jedoch nicht nur bereitwillig diese solitäre Position abseits der akademischen Institutionen, sondern geht noch einen Schritt weiter, um eine Monopolisierung seines Archivs voranzutreiben. Frei nach dem ersten Gebot der Quellenstudien GmbH (»Ich bin der His-

33 Zum Vorgang der geeigneten Auswahl der Lebenspartnerin bei Sigmund Freud vgl. Klaus Theweleit: Objektwahl. (All You Need Is Love... ). Über Paarbildungsstrategien. Bruchstück einer Freudbiographie, München 1996. 
toriker der Technik und Du sollst keine anderen Technikgeschichten neben mir haben«), betreibt Feldhaus vehement den Ausbau der Alleinherrschaft seines Unternehmens, um gleichzeitig die aufkeimende Konkurrenz in Gestalt von Conrad Matschoß und dem VDI ebenso ausdauernd wie systematisch zu bekämpfen. Sein Ziel besteht mithin darin, diese Konkurrenz wissenschaftlich zu desavouieren. Als eindruckvollster Beleg für diese Monopolisierungsbestrebungen können seine über viele Jahre vorgebrachten Anschuldigungen gegen den VDI dienen, die in der Geschichte der wissenschaftlichen Dispute ihresgleichen suchen. Die Auseinandersetzung vermag ohne Schwierigkeiten neben so berühmten Kontroversen bestehen, wie sie etwa Robert Hooke entfacht hat, ${ }^{34}$ oder aber neben dem Streit zwischen Jean François Champollion und Thomas Young um das Verdienst einer ersten Entzifferung der Hieroglyphen, die ihrerseits nicht vor seitenlangen Beschimpfungslitaneien gegen den Kontrahenten in ihren jeweiligen Publikationen zurückschreckten.

Neben diesem (lebensnotwendigen) Monopolisierungsprozess bleibt eine weitere Leistung von Feldhaus bemerkenswert. Es gelingt ihm, infolge geschickter Werbemaßnahmen einen vollkommen neuen Markt zu schaffen und nachhaltig zu explorieren, und zwar für das bis dato eher randständige Genre der Firmenschriften. Im System seiner Aufzeichnungen werden auch die sog. Tages- bzw. Jahresdaten-Karteien gepflegt, also Hinweise auf Artefakte und ihre Ursprünge kalendertechnisch aufbereitet, chronologisch geordnet, so dass etwaige Jubiläen von Gegenständen, deren Geburtstage oder sonstige Feierlichkeiten künftig von ihren Herstellern zu begehen wären, den Produzenten im Vorfeld lange vorher angekündigt werden können, um ihnen gleichzeitig das Angebot für eine passende Firmenschrift zu unterbreiten.

$\mathrm{Zu}$ fragen bleibt nun, ob seine generelle Strategie der Wissensproduktion als Verfahren und ob Feldhaus als Historiograph der Technik einem bestimmten Typus gehorcht. Was ist das Charakteristische an dieser wissenschaftlichen Form und dem zweifelsohne wagemutigen Versuch, in einem Zeitalter nahezu unüberschaubarer Innovationen von Industrie und technischer Forschung die Universalgeschichte eben jener Wissensgebiete möglichst vollständig zu verzeichnen?

Zwei theoretische Beschreibungsmuster bieten sich bei dieser Frage an, denen beiden wiederum die gleiche Denkfigur zu Grunde liegt und die hier noch in aller Kürze als Diskussionsangebot skizziert sei. Man könnte zunächst im Anschluss an Michel Serres den >Nestor der Technikgeschichtsschreibung`, Franz Maria Feldhaus, als Parasiten beschrei-

34 Vgl. dazu Bernhard Siegert: Passage des Digitalen. Zeichenpraktiken der neuzeitlichen Wissenschaften 1500-1900, Berlin 2003. 
ben. Unmittelbar einsichtig ist dieses Handlungs- und Kommunikationsschema hinsichtlich der Art und Weise, wie Feldhaus durch seine Familienproduktion die Bruchstücke der Technikgeschichte auf Karteikarten versammelt, um sie rekombiniert und mit vereinten Kräften zu neuen Texten zu ordnen und damit die Erträge seiner Arbeit zu sichern. Keine Zeile verläßt das Archiv in Richtung Druckerpresse, an der nicht das versammelte >Harem mit Lieblingsfrauen` die zarten Hände angelegt hätte.

Feldhaus wacht über den textuellen Strom der Karteiarbeit, er sitzt an den Kreuzungen und Schnittstellen der einzelnen Textbausteine, er leitet den Strom der technikhistorischen Fragmente auf Karteikarten und differenziert, unterscheidet, lenkt, gleich einem Weichensteller wie dem Maxwell'schen Dämon, die Elemente, die so zu dem jeweils gewünschten Artikel zusammenfinden. Die Prozessierung der Daten verrichten die Frauen. Seine Tätigkeit besteht in der Supervision. Er ist der Diktator.

Bei dieser Beschäftigung mit Technik geschieht nun ein kategorialer Wechsel. Feldhaus rezipiert die Baupläne und Patentschriften der Maschinen und Objekte nicht etwa, um daraus wiederum neue Maschinen oder Apparate herzustellen. Sein Produkt besteht statt dessen aus kaum gegenständlicher Information, aus rein geschichtlicher Beschreibung. Die Metamorphose von der Technik zur Technikhistoriographie zwingt ihn, vom »Ingenieur«, der er so gerne gewesen wäre, zum Geschichtenschreiber zu mutieren. Der Parasit, so Serres, kreuzt die herkömmliche Logik, weil er den konventionalisierten Austausch, hier also die diskursive Kontinuität technischer Entwicklungen aufhebt, indem er die Währung wechselt. »Er versucht Stimme gegen Substanz zu tauschen, Luftiges gegen Solides oder Superstruktur gegen Infrastruktur. [...] er erfindet etwas Neues. $\ll^{35}$ Die Innovation liegt dabei nicht zuletzt in der Erkenntnis, dass mit der Verbreitung von Technikgeschichten statt Blaupausen oder Bauanleitungen ebenso einträglich ein Auskommen erreicht werden kann. Der >Ingenieur`steht also fortan nicht mehr in der ausschließlichen Verlegenheit, Apparate zu entwerfen und zu bauen, sondern das Wissen um die Konstruktion und Entstehung selbst gerät zum profitablen Produkt. ${ }^{36}$ »Der Parasit erfindet etwas Neues. Er eignet sich eine Energie an und bezahlt sie mit Informationen. Er eignet sich den Braten an und zahlt mit Geschichten. « ${ }^{37}$

Genau diesem Schema folgt Dr. Ing. h.c. Franz Maria Feldhaus, wenn er einen bislang unbekannten Markt eröffnet für Firmenschriften, aber auch für Auftragsarbeiten von Unternehmen, mit gezielten Recher-

35 Michel Serres: Der Parasit, Frankfurt a. M. 1987, S. 58.

36 Spätestens an diesem kategorialen Wechsel erfolgt die manifeste Einladung zur Betriebs- und Wirtschaftsspionage.

37 M. Serres: Der Parasit, S. 59f. 
chen offene Fragen zu bestehenden oder abgelaufenen Patenten zu klären. Feldhaus ist der Parasit der Technik, indem er alle verfügbaren Informationen versammelt, um sie dann (teuer) an jene zu verkaufen, die ihrer bedürfen. Und weil dieser informationelle Mangel, die Notwendigkeit zur fundierten Information nicht immer gleich einzusehen ist, muß Feldhaus diesen Bedarf mitunter selbst erst noch wecken, beispielsweise mit der kalkulierten Konstruktion von Jubiläen.

Um 1900 hat sich in der sog. industriellen Forschung und Entwicklung ein leistungsfähiger technischer Diskurs eingestellt: Unternehmen beschäftigen Ingenieure, die geschult an renommierten technischen Hochschulen die Kenntnisse und Theoreme aus ihrer Ausbildung in der Unternehmenspraxis von Fertigung und Maschinenbau weiterentwickeln, Erfahrungen anhäufen, in Fachorganen wie etwa dem Polytechnischen Zentralblatt oder Patentschriften distribuieren, um so das Wissen um die Konstruktion von Maschinen oder Anlagen rückzukoppeln an die Stätten ihrer Ausbildung. Ein geschlossener Regelkreis mit oszillierenden Sender- und Empfängerpositionen, die jedoch jederzeit eindeutig zuordnungsfähig bleiben. Wo ist hier der Parasit, der Dritte in der zweiwertigen Logik und Kommunikation? Der Dritte muß erst noch gesucht werden, muß sich möglicherweise - wie im Fall Feldhaus - selbst erschaffen, er ist vorerst verborgen, unsichtbar. Ein Dritter ist also ausgeschlossen, zunächst, wenn er nicht diesen kategorialen Wechsel vom technischen hin zum technikhistoriographischen Diskurs vollzieht. Dann ist er zwar noch nicht gleich in das System eingeschlossen, nichtsdestoweniger jedoch, so die Logik von Serres, Bestandteil dieses Systems, und ein produktiver Mehrwert fällt für den Parasiten, den nunmehr eingeschlossenen ausgeschlossenen Dritten ebenfalls ab. Denn schließlich profitieren die Unternehmen von ihrer eigenen Historizität, und zwar zum einen beispielsweise durch ihre Firmenschriften als Gedächtnisfunktion der historischen Genese von Gegenständen, aus deren einmal aufgezeichneter Entwicklung sich möglicherweise wiederum Verbesserungen des Produkts ableiten lassen. Zum anderen dient die Firmenschrift selbstverständlich auch als geeignete Werbemaßnahme, um auf die Produkte des Unternehmens aufmerksam zu machen. Von diesem Profit profitiert seinerseits der Parasit und läßt sich dies entsprechend honorieren.

Analog zu dieser Denkfigur des eingeschlossenen ausgeschlossenen Dritten verhält sich auch ein anderer Typus, der hier als Kontrast und komplementäre Beschreibung zum Parasiten nicht unerwähnt bleiben 
soll. »Definition: der Dilettant ist der produktive Nicht-Fachmann, einer, der das Metier oder das Handwerk nicht beherrscht, und doch etwas produzieren möchte. $\ll^{38}$ Feldhaus, der gescheiterte Jesuitenschüler, der Artistengehilfe, Handlungsreisende, Elektroinstallationshilfsarbeiter und Autor von Gelehrtengräberschriften beginnt sich um die Jahrhundertwende 1900 Ingenieur zu nennen, da diese Berufsbezeichnung weder durch einen Berufsverband geschützt noch von einer Ingenieurszunft vereinnahmt ist. Die Zugehörigkeitserklärung kann noch kraft eigener Bestimmung erfolgen, die Würde dieses Titels muß nicht von einer Institution verliehen werden. So eignet sich Feldhaus einen Namen an, entlehnt ihn einem technischen Diskurs, an dem er gar nicht teilhaben wird. Ein Kommunikationskanal dient als Taufpate, den er zumindest als Sender, das heißt als Entwickler von technischen Objekten, nie bedienen wird. Dennoch nennt er sich >Ingenieur<. Dilettantismus setzt eine sehr fundamentale Poetik voraus, die sich grundlegende Fragen nach Lesen, Schreiben und Sprechen, nach den basalen Kulturtechniken stellt. »Man weiß nicht, wie man lesen, man weiß nicht, wie man sprechen kann, und deshalb muß man mit den einfachen Dingen anfangen, klein anfangen, ganz unten. $\ll^{39}$ Der Dilettant ist also zunächst sprachlos, ohne zuhörende Öffentlichkeit, ohne Anleitung oder Methode, aber mit einem Vorhaben vor Augen, einem Projekt im Kopf, das es zu realisieren gilt. Wie anfangen? Das ist die dringende Frage eines jeden Neulings - und zudem der analytische Ansatzpunkt einer Dilettantismus-Forschung. ${ }^{40}$ Im Jahr 1900 beginnt Franz Maria Feldhaus, sein Archiv aufzubauen mit einer Sammlung von Daten und kleinen Textbausteinen, die »irgendwie technischhistorisch $\aleph^{41}$ aussehen und die sich mit der Zeit, so der spätere Plan, zu einer Weltgeschichte der Technik verdichten und vervollständigen sollen. »Der Dilettant ist derjenige, der den Teil für das Ganze nimmt. [...] Er ist zudem jemand, der keine halben Sachen macht, sondern Halbes für Ganzes hält oder halten will. $\aleph^{42}$ Nochmal die Frage: Wie anfangen? Was ist die Poetik der Historiographie? Wie funktioniert die Technik der noch

38 Erhard Schüttpelz: »Die Akademie der Dilettanten«, in: Stefan Dillemuth (Hg.), Akademie, Köln 1995, S. 40-57, hier S. 40.

39 Vgl. Georg Stanitzek: »Über Professionalität«, in: VERSTÄRKER (1997), Bd. 2, Nr. 3, www.culture.hu-berlin.de/verstaerker/vs003/, o.S.

40 Vgl. E. Schüttpelz: »Die Akademie der Dilettanten«, S. 40-57, G. Stanitzek: »Über Professionalität«, und Volker Pantenburg: »Dreizehn Buchstaben kennen. Trotzdem sprechen. Trotzdem schreiben. Über Dilettantismus«, in: Weimarer Beiträge, Jg. 47, Nr. 2, S. 241-255.

41 F. M. Feldhaus: »Archiv Geschichte d. Technik, e.V.«, S. 158.

42 V. Pantenburg: »Dreizehn Buchstaben kennen. Trotzdem sprechen. Trotzdem schreiben. Über Dilettantismus«, S. 243. 
nicht ausgebildeten Technikgeschichtsschreibung? Man denke jetzt einfach für einen kurzen Moment, ein `Text sei >Musikı, man lese also stets >Text८, wenn im Folgenden >Musik « steht: »Es ist seine Technik, Musik aus Fertigteilen zu konstruieren. [...] Dabei gibt es keine feste Reihenfolge für die Elemente, kein logisches Eins-ans-andere-Reihen, sie sind austauschbar, wiederholbar, annähernd jedes mit jedem zu koppeln, beliebig $\mathrm{zu}$ zerschneiden, in Bruchstücken verwendbar. ${ }^{43}$ Was Ornella Volta dem jungen Erik Satie - nach Volker Pantenburg ein Dilettant par excellence - konstatiert, ist reine Karteikastentechnik, die Verschaltung beweglicher (textueller) Einheiten zu etwas Neuem, zu einem synthetisierten Ganzen. Das Besondere der Dilettanten-Arbeit ist ein spezifisch artistisches Element: die Arbeit mit Fragmenten, Stücken, Satz für Satz, Einstellung für Einstellung, Fakt für Fakt, eine eigenartige Handarbeit, ${ }^{44}$ die eigentlich ein simples, weil naheliegendes Handwerk darstellt. Da eine allgemeine Sprache immer schon vorhanden ist, genügt es deshalb lediglich zu zitieren: »das Eigene entsteht nur in der buchstäblichen Auseinandersetzung der und mit den fremden Vorgaben«, wie Georg Stanitzek das Verfahren des Dilettanten kennzeichnet. ${ }^{45}$

Wie kann Feldhaus mit seiner Forschung beginnen, wenn er weder im Regelwerk des Ingenieurs noch in der Methodik des Historiographen geschult ist? »Die Dilettanten vergessen nie und erinnern mit RegelmäBigkeit daran, daß im Zentrum allen relevanten Know-hows die Autodidaxie sitzt $~^{46}{ }^{46}$ Aus dem Mangel an Ausbildung, dem fehlenden Handwerkszeug erwächst zwangsläufig die Notwendigkeit zur Innovation. Das Neue ist also unvermeidlich dilettantisch, weil die alten, einvernehmlich akzeptierten Standards nicht greifen; erst nach einer gewissen Latenzzeit wird die zuvor als >dilettantisch < denunzierte Innovation dann vom Fach entweder kanonisiert oder aber geflissentlich übersehen. ${ }^{47}$

Der Dilettant wendet sich mit seinem Versuch der Innovation gegen etwas, gegen eine Barriere; er steht quer zu einem etablierten Kommunikationskanal, zum anerkannten Diskurs mit seinem festgelegten Regelwerk, der von den Eingeweihten und Berufenen, von den Professionellen

43 Ornella Volta: »Anmerkungen«, in: dies. (Hg.), Erik Satie. Schriften, 3. Aufl., Hofheim, S. 19, zit. nach V. Pantenburg: »Dreizehn Buchstaben kennen. Trotzdem sprechen. Trotzdem schreiben. Über Dilettantismus«, S. 241-255.

44 Wie die sog. Centontendichtung; Cento ist ein Gedicht aus Fetzen, im 4. Jahrhundert von Proba erstmals durchgeführt, vgl. G. Stanitzek: »Über Professionalität«.

45 G. Stanitzek: »Über Professionalität«.

46 Ebd.

47 Vgl. E. Schüttpelz: »Die Akademie der Dilettanten«, S. 42. 
betrieben wird. Die Teilnehmer an diesem Diskurs schließen den Dilettanten aus von einer, von ihrer eigenen Produktion, zumal wenn dieser Produktion nicht einmal eine Rezeption ihrer diskursiven Fachverhandlungen vorangeht. Damit findet sich der Dilettant in der Position des ausgeschlossenen Dritten wieder, dessen Beiträge zur gelehrten Kommunikation keine Beachtung finden, nicht finden dürfen, da seine Sprecherposition die eines Unberufenen ist. Die hartnäckige Ignoranz des VDI gegenüber Feldhaus legt darüber deutlichstes Zeugnis ab. Und doch befindet sich in dieser Barriere eine Lücke, eine Durchlässigkeit, ein blinder Fleck innerhalb der strengen Diskursregelung, durch die ein Fortschritt, ein Impuls, eine Innovation erfolgen kann. Denn die vereinbarten Standards sind blind für Innovationen, weil ihre Aufgabe darin besteht, alle unangemessenen Eingaben auszuschließen. »Dilettantismus ist mit gleichem Recht, und zwar vor allem in der Blindheit der Innovation, eine Produktionskategorie für Rezipienten, eine produktive Kategorie, mit deren Hilfe man etwas Anderes, und manchmal auch etwas Neues schaffen kann. ${ }^{48}$ Aus diesem Grund trägt der ausgeschlossene Dritte maßgeblich zur Erhaltung und Funktionsfähigkeit des Systems als Ganzem bei. Zwar faßt man Feldhaus' Einwürfe zumeist als unliebsame Störungen auf, man blendet sie daher weitestgehend aus. ${ }^{49}$ Doch ohne diese Irritationen droht sich der technikhistorische Diskurs trotz seiner vergleichsweise jungen Existenz bereits wieder zu erschöpfen. Franz Maria Feldhaus, der unsichtbare Dritte, wird damit zum unabdingbaren Bestandteil des Systems. Denn gerade als Paria, als unerwünschte Person, findet er erneut Anschluss an das System Technikgeschichte, das ihn gewissermaßen aus systemischen Gründen der Selbsterhaltung notwendigerweise wieder einbezieht.

Nun könnte man fragen, was mit der Erkenntnis anzufangen sei, dass man Franz Maria Feldhaus möglicherweise nicht nur als Parasiten, sondern auch als Dilettanten einzuordnen weiß. Entscheidend in diesem Zusammenhang ist das Charakteristikum Dilettantismus als solches. Denn seine Präliminarien und funktionalen Zusammenhänge können belegen, dass dem Dilettanten nicht nur spezifisch neue Erkenntnisleistungen zu Gute kommen, sondern gleichfalls eine besondere Innovationskraft innewohnt. Es ist dieser Dilettantismus, der als eine der wesentlichen Kategorien anzusehen ist, mit denen die produktiven Eigenschaften jener Projektemacher, Visionäre und Lebensreformer zu charakterisieren sind, wie sie im Fin de siècle davon ausgehen, mit ihren Ideen die Welt verändern zu können. Dilettantismus ist demnach eine der Motivationen und

48 Ebd., S. 53f.

49 Seit 1925 taucht der Name >Feldhaus` in keiner Publikation mehr auf, die in entferntester Kooperation mit dem VDI entsteht. 
Triebkräfte, die maßgeblich dazu ermutigen, mit einer schlichten Idee gleich die ganze Welt (hier: der Technik) einfangen und umfassen zu können. ${ }^{50}$

50 Vgl. dazu M. Krajewski: Restlosigkeit. Weltprojekte um 1900. 